\title{
Long-term effects of alendronate on fracture healing and bone remodeling of femoral shaft in ovariectomized rats
}

\author{
Ling-jie $\mathrm{FU}^{1,2}$, Ting-ting $\mathrm{TANG}^{1}$, Yong-qiang $\mathrm{HAO}^{1}$, Ke-rong DAI ${ }^{1,2, *}$ \\ ${ }^{1}$ Department of Orthopedic Surgery, Ninth People's Hospital, Shanghai Jiao Tong University School of Medicine, Shanghai 200011, \\ China; ${ }^{2}$ Orthopedic Cellular and Molecular Biology Laboratory, Institute of Health Sciences, Chinese Academy of Sciences, and \\ Shanghai Jiao Tong University School of Medicine, Shanghai 200011, China
}

\begin{abstract}
Aim: To investigate the long-term effects of alendronate (Aln), a widely used oral bisphosphonate, on fracture healing and bone remodeling in ovariectomized rats.

Methods: Adult female SD rats underwent ovariectomy, and then bilateral femoral osteotomy at 12 weeks post-ovariectomy. From d 2 post-ovariectomy, the animals were divided into 3 groups, and treated with Aln ( $\left.3 \mathrm{mg} \cdot \mathrm{kg}^{-1} \cdot \mathrm{d}^{-1}, \mathrm{po}\right)$ for 28 weeks (Aln/Aln), Aln for 12 weeks and saline for 16 weeks (Aln/Saline) or saline for 28 weeks (Saline/Saline). At 6 and 16 weeks post-fracture, the fracture calluses were examined with X-ray radiography, and biomechanical testing and histological analysis were performed. The calluses were labeled with tetracycline and calcein to evaluate the mineral apposition rate (MAR).

Results: The fracture line was less distinct in the 2 Aln-treated groups at 6 weeks post-fracture, and disappeared in all the 3 groups at 16 weeks post-fracture. The size of the callus and radiographic density of the femora in the Aln/Aln group were the highest among the 3 groups at 6 and 16 weeks post-fracture. Similar results were observed in the ultimate load at failure and energy absorption. However, the treatment with Aln delayed endochondral ossification of the callus, and significantly increased the total sagittal-sectional area, percentage callus area and callus thickness, and decreased the MAR at 6 and 16 weeks post-fracture.

Conclusion: In the ovariectomized rat model, Aln is beneficial for the mechanical properties of the callus, but delays callus remodeling by suppressing the remodeling of woven bone into lamellar bone.
\end{abstract}

Keywords: alendronate; bisphosphonate; ovariectomized rat; fracture healing; callus remodeling; osteoporosis

Acta Pharmacologica Sinica (2013) 34: 387-392; doi: 10.1038/aps.2012.170; published online 4 Feb 2013

\section{Introduction}

Osteoporosis is a major health problem characterized by compromised bone strength that predisposes patients to an increased risk of fracture ${ }^{[1,2]}$. Pharmacologic agents that modulate bone formation and bone remodeling, such as bisphosphonates (BP), parathyroid hormone, estrogen, selective estrogen receptor modulators, calcitonin, and vitamin $\mathrm{D}$, are in broad use and development for the treatment of osteoporosis and other disorders of bone fragility ${ }^{[3,4]}$. When choosing an osteoporosis treatment drug, it is important to consider fracture healing because osteoporotic patients undergoing BP treatment may suffer fragility fractures ${ }^{[5,6]}$. In addition, clinicians have been concerned that fractures do not heal properly in individuals exposed to BP treatments, which have

\footnotetext{
* To whom correspondence should be addressed.

E-mail krdai@163.com

Received 2012-07-29 Accepted 2012-11-15
}

the unique characteristic of accumulating in the skeleton ${ }^{[7]}$. Therefore, there is significant interest in the long-term effect of $\mathrm{BP}$ therapy on bone repair and whether the accumulation of $\mathrm{BP}$ in the skeleton is detrimental to fracture healing.

BP differ in their effect on bone growth, remodeling, mineralization, incidence of fracture, and repair. These differences are related to the chemical structure of the compounds, their potency, dosage levels, route of administration, treatment period, and rates of remodeling before treatment ${ }^{[8]}$. In the present study, ovariectomized rats, which are recommended by the Food and Drug Administration (USA) as a model of postmenopausal osteoporosis, were used. Alendronate (Aln), a widely used oral BP, was selected as the experimental agent; a dosage of $3 \mathrm{mg} \cdot \mathrm{kg}^{-1} \cdot \mathrm{d}^{-1}$ (corresponding to $10 \mathrm{mg} / \mathrm{kg}$ for a $60-\mathrm{kg}$ man) was selected because it is the common dosage used by osteoporotic patients ${ }^{[9]}$. To our knowledge, this is the first study to evaluate the long-term effect of Aln administration on fracture 
healing and bone remodeling in ovariectomized rats.

\section{Materials and methods Materials}

Commercial Aln was purchased from Mosadong, USA, and dissolved in saline vehicle. Aln $\left(3 \mathrm{mg} \cdot \mathrm{kg}^{-1} \cdot \mathrm{d}^{-1}\right)$ was administered by oral gavage, which corresponds to the clinical dose of approximately $10 \mathrm{mg} / \mathrm{d}$ for a $60-\mathrm{kg}$ human ${ }^{[9]}$.

\section{Animals and experimental design}

Forty-five female Sprague-Dawley (SD) rats aged six months $(260 \pm 3 \mathrm{~g})$ were purchased from Shanghai Jiaotong University School of Medicine (Shanghai, China) and acclimated for 2 weeks to the local vivarium conditions $\left(24 \pm 2^{\circ} \mathrm{C}\right.$ and 12 -h lightdark cycle). During the experimental period, animals were housed in cages $\left(988 \mathrm{~cm}^{2}\right.$ in floor area and $18 \mathrm{~cm}$ in height) and allowed free access to water and pelleted commercial rodent diet. The experimental protocol was approved by the Institutional Animal Care and Use Committee of the Shanghai Jiao Tong University Animal Study Committee.

The animals were randomly allocated into 3 groups (Saline/Saline, Aln/Saline, and Aln/Aln) and given an ovariectomy at the beginning of the study (Figure 1). The Aln/ Aln group received Aln for 12 weeks before being subjected to a standardized femoral fracture and during the fracture healing period of 6 and 16 weeks. The Aln/Saline group received Aln before the fracture and saline during the healing period. The Saline/Saline group received saline during the experiment.

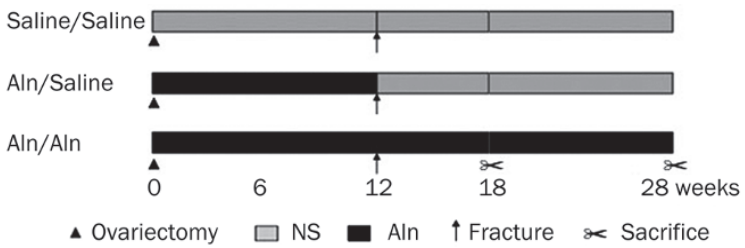

Figure 1. Experimental design showing the different treatment groups, time point of euthanasia, and agents used in different groups. The Aln/Aln group received Aln for 12 weeks before being subjected to a standardized fracture of the femora and during the fracture healing period for 6 and 16 weeks. The Aln/Saline group received Aln before the fracture and saline during the healing period. The Saline/Saline group received only saline during the experiment.

General anesthesia for all operative procedures was achieved by initial subcutaneous administration of $0.05 \mathrm{mg} / \mathrm{kg}$ atropine and $1.5 \mathrm{~mL} / \mathrm{kg}$ fentanyl-fluanisone (Hypnorm, Janssen Pharmaceuticals, Oxford, UK) followed by an intraperitoneal injection of $20 \mathrm{mg} / \mathrm{kg}$ pentobarbital sodium. Buprenorphine $(0.45 \mathrm{mg} / \mathrm{kg})$ (Temgesic, Reckitt \& Cloman, Hull, UK) was administered subcutaneously for postoperative analgesia. In addition, the animals were given one intraperitoneal injection of $70 \mathrm{mg}$ of streptomycin to prevent infection.

All rats were given bilateral ovariectomy at the beginning of the study. Rats were placed under general anesthesia under aseptic conditions. A single mid-dorsal incision was made through the shaved skin and fascia. Both ovaries were identified, clamped, and removed. The muscle layer and skin were subsequently sutured with polyglactin absorbable sutures. Animals were caged individually and allowed to recover under a heat lamp/heat mat for a minimum of $24 \mathrm{~h}$ postovariectomy. Twelve weeks later, when the animals were nine months of age, a bilateral femoral osteotomy was performed, as previously described ${ }^{[10-14]}$. Briefly, a transverse osteotomy was made at the midshaft of the bilateral femora, the fracture fragments were contacted and stabilized, and the intramedullary was fixed with a stainless wire (diameter $1.5 \mathrm{~mm}$ ). Wire was cut on the surface of the intercondylar groove to avoid restricting the motion of the knee joint. Unrestricted activity was allowed after recovery from anesthesia. Body weights were measured weekly, and treatment dosages were adjusted accordingly. The rats were euthanized at 6 and 16 weeks after the osteotomy by exsanguination from the abdominal artery under general anesthesia, as described above. Animals received an intraperitoneal injection of tetracycline and calcein on days 12 and 2 before euthanasia at 16 weeks post-fracture to determine the mineral apposition rate (MAR).

The animals resumed normal activity within a few days after the osteotomy. Among 45 animals at the beginning of the study, 3 were excluded due to failure of fixation or postsurgical infection.

\section{Soft X-ray radiography}

Briefly, bilateral femora were excised and dissected from soft tissues, and the intramedullary wires were extracted. Soft $\mathrm{X}$-ray radiographs of the femora were taken anteroposteriorly $\left(26 \mathrm{kVp}, 12 \mathrm{~s}\right.$; Faixtron, Japan) ${ }^{[15]}$. The image was quantitatively analyzed by NIH Image Analysis software.

\section{Biomechanical testing}

A three-point bending test was carried out on the fractured calluses after X-ray image acquisition using a mechanical testing machine (Lloyd EZ20, England). The femur was placed with its anterior surface facing upward on two lower support bars $15 \mathrm{~mm}$ apart, and the loading bar was positioned at the central part of the fracture site (anteroposterior position). A compression load was applied at a rate of $2.0 \mathrm{~mm} / \mathrm{min}$ until breakage. The ultimate load at failure $(\mathrm{N}$; maximum force that the specimen sustained) and energy absorption (mJ) were calculated.

\section{Histology}

For histological analyses, the samples were fixed in phosphate buffered formalin for $48 \mathrm{~h}$, followed by a sagittal cut through the fracture site using a low speed saw with a diamond disc blade (Buehler Ltd, Lake Bluff, IL, USA). One portion was decalcified in $0.5 \mathrm{~mol} / \mathrm{L}$ ethylenediaminetetraacetic acid ( $\mathrm{pH} 7.4)$ for $21 \mathrm{~d}$. After dehydration with a series of ethanol rinses and a rinse with xylene, the specimens were embedded in paraffin. Five-micron-thick sections were 
cut and stained with hematoxylin and eosin (HE) and evaluated qualitatively by light microscopy (Zeiss Aixoplan with Spot RT digital camera, Zeiss, German). The image was quantitatively analyzed by Image Pro Plus Software (Media Cybernetics, Silver Spring, MD). The total sagittal-sectional area $\left(\mathrm{T}\right.$.Ar; $\left.\mathrm{mm}^{2}\right)$, callus area $\left(\mathrm{Cl}\right.$.Ar; $\left.\mathrm{mm}^{2}\right)$, and callus thickness $(\mathrm{Cl}$.Th; $\mu \mathrm{m})$ were measured at $40 \times$ magnification. These measurements were used to calculate the percentage callus area $(\% \mathrm{Cl} . \mathrm{Ar}=\mathrm{Cl} . \mathrm{Ar} \times 100 / \mathrm{T} . \mathrm{Ar} ; \%)$.

\section{Determination of mineral apposition rate (MAR)}

To evaluate the MAR, fluorescent bone labels were administered. Briefly, each rat was given an intraperitoneal injection of tetracycline ( $15 \mathrm{mg} / \mathrm{kg}$, Sigma, USA) and calcein (10 $\mathrm{mg} / \mathrm{kg}$, Sigma) on days 12 and 2, respectively, before being euthanized at 16 weeks post-fracture. After the animals were euthanized, the specimens were fixed in $10 \%$ formalin solution and embedded in methyl methacrylate. All the tissues were cut lengthwise using a circular water-cooled diamond saw. The sections were visualized and photographed with an E-800 fluorescent microscope (Nikon). The mean tetracycline (yellow) and calcein (green) double-labeling interval was determined by the Zeiss Axioplan Imaging System. The interval was divided by the administration time interval $(10 \mathrm{~d})$, and the mean value is the $\operatorname{MAR}(\mu \mathrm{m} / \mathrm{d})$.

\section{Statistical analysis}

All data are expressed as the mean \pm standard deviation (SD) and were analyzed with SPSS (Statistical Package for the Social Sciences) 10.0 (SPSS, Chicago, IL, USA). One-way ANOVA with Bonferroni's post test (multiple comparisons) and Student-Newman-Keuls tests (comparisons between two groups) were used as appropriate. Differences were considered as statistically significant for $P$ values $<0.05$.

\section{Results}

\section{Soft X-ray radiography}

X-ray images (Figure 2A) revealed the fracture line in all groups at 6 weeks post-fracture; however, it was less distinct in the Aln groups. In addition, both Aln groups had larger calluses than the control group at the same time point. At 16 weeks post-fracture, the fracture line was invisible in all study groups. Furthermore, the calluses in the Aln groups were larger than those in the control group but smaller than the calluses at 6 weeks post-fracture. Of all the calluses in the study groups, the Aln/Aln group had the largest calluses at both end points.

The femora in the Aln groups had a greater radiographic density than the control group $(P<0.01)$, with the highest density in the Aln/Aln group, suggesting that treatment by Aln has a positive effect on osteoporosis (Figure 2B and 2C).

\section{Biomechanical testing}

As shown in Figure 3A and 3B, the ultimate load at failure of the Aln groups was higher than those of the control group at 6 weeks post-fracture, and the energy absorption in the Aln

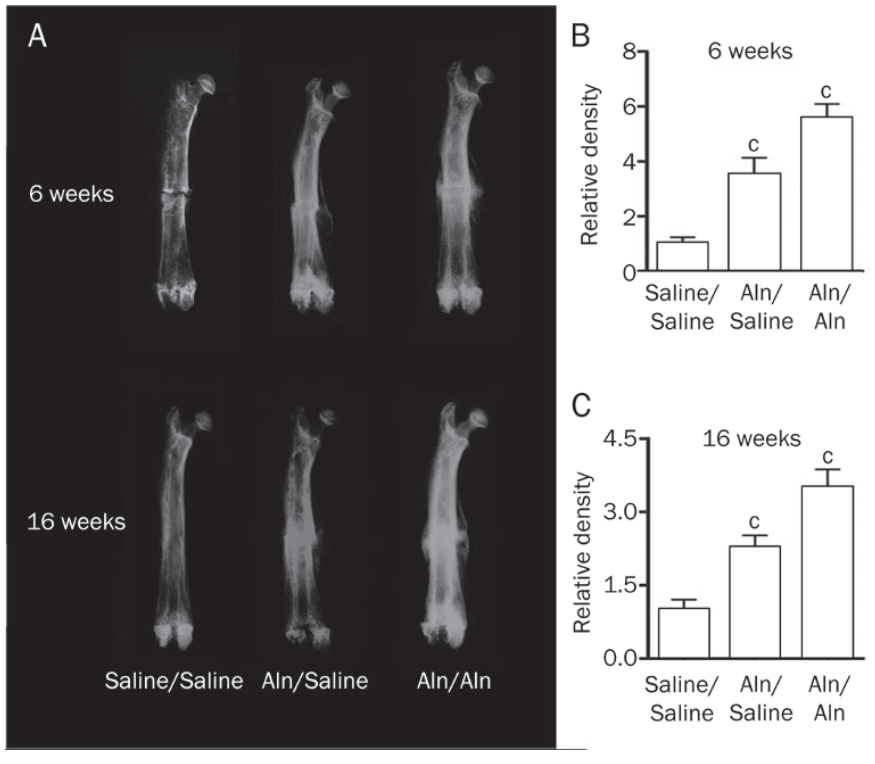

Figure 2. Soft X-ray radiographs of fractured femora. (A) The fracture line was visible in the control group at 6 weeks post-fracture, while it was invisible in all groups at 16 weeks post-fracture. (B and $C$ ) The relative density was quantitated and statistically analyzed $\left({ }^{c} P<0.01\right.$ vs Saline/Saline).
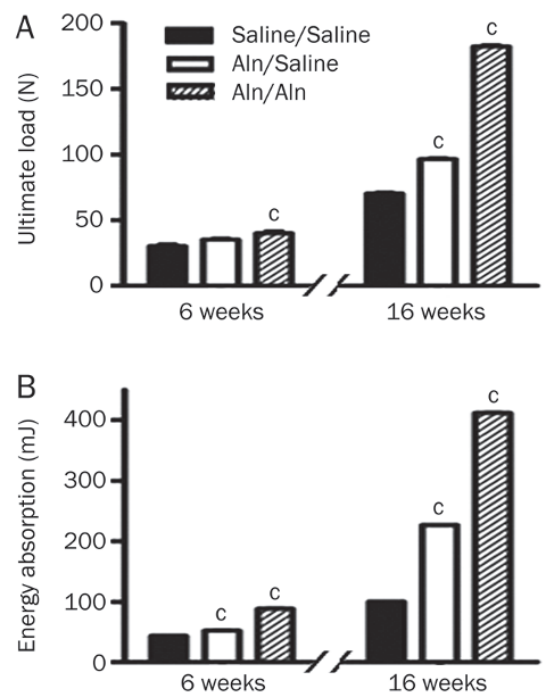

Figure 3. Biomechanical properties of fractured calluses in the three treatment groups. The data are expressed as the mean \pm SD $\left({ }^{\circ} P<0.01\right.$ vs Saline/Saline, $n=6$ ). (A) Ultimate load; (B) Energy absorption.

groups was higher than that in the control group $(P<0.01)$. At 16 weeks post-fracture, the ultimate load and energy absorption in the Aln groups were also higher than those in the control group $(P<0.01)$. At both end points, the ultimate load and energy absorption in the Aln/Aln group were the highest. These results demonstrate that oral gavage of Aln could strengthen the mechanical properties of the femora in ovariectomized rats. 


\section{Histology}

Sections obtained from the Aln groups at 6 weeks post-fracture (Figure 4) showed an immature hyaline cartilage-like region in the fracture gap, although partially woven bone persisted in the healing callus of the control group, suggesting a delay in endochondral ossification by Aln. At 16 weeks post-fracture (Figure 4), the healing fracture callus of the Aln groups mainly consisted of woven bone, remodeling toward mature lamellar bone. In contrast, the remodeling of the callus in the control group was nearly finished, and the callus seemed to be normal.
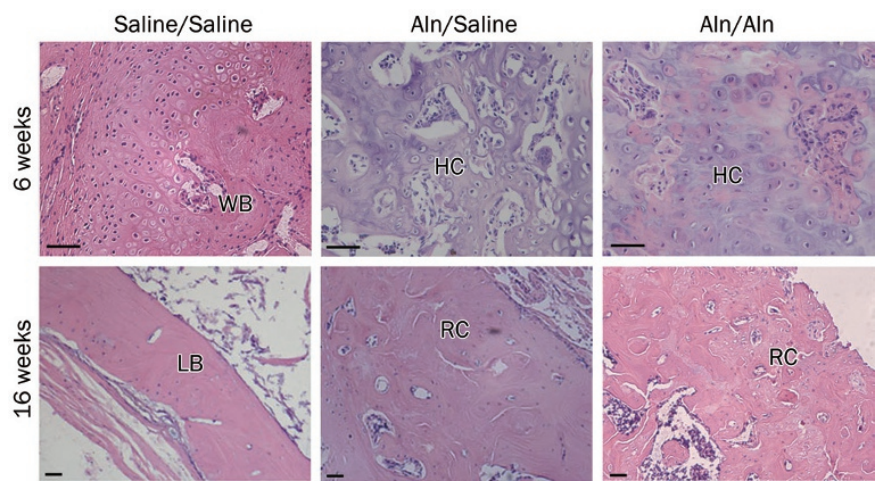

Figure 4. Histological changes in fracture healing at 6 and 16 weeks after osteotomy. At 6 weeks post-fracture, in the control group, mature woven bone (WB) had more integrity, and the callus was thick and continuous. In the Aln groups, the calluses primarily consisted of hyaline cartilage $(\mathrm{HC})$. At 16 weeks post-fracture, the healing fracture callus remodeled better in the control group than the Aln groups. LB: lamellar bone, RC: remodeling callus. Scale bar represents $25 \mu \mathrm{m}$.

Quantitative analysis showed that at 6 weeks post-fracture, the T.Ar of the femur was larger in the Aln/Saline group $\left(63.54 \pm 4.71 \mathrm{~mm}^{2}\right)$ and the Aln/Aln group $\left(86.08 \pm 5.54 \mathrm{~mm}^{2}\right)$ compared with the Saline/Saline $\left(44.93 \pm 1.56 \mathrm{~mm}^{2}\right)(P<0.01)$ (Figure 5A). Furthermore, at 16 weeks post-fracture, the T.Ar of the femur in the Aln groups was higher than that in Saline/ Saline $(P<0.01)$ (Figure 5A). The Cl.Th and percentage callus area were also larger in the Aln groups compared with the Saline/Saline group at both end points $(P<0.01)$ (Figure 5B and 5C). In addition, T.Ar, $\mathrm{Cl}$.Th and percentage callus area in the Aln/Aln group were the highest among the three groups.

\section{Mineral apposition rate}

Representative fluorescence images of the fracture calluses are shown in Figure 6. The Aln/Saline group attained a significantly lower rate of calcification $(5.14 \pm 0.32 \mu \mathrm{m} / \mathrm{d})$ than the Saline/Saline group $(5.82 \pm 0.42 \mu \mathrm{m} / \mathrm{d})(P<0.05)$ (Figure 5D). In addition, continued Aln treatment post-fracture further decreased the MAR in the Aln/Aln group $(4.60 \pm 0.36 \mu \mathrm{m} / \mathrm{d})$ compared with control $(P<0.01)$ (Figure 5D).
A

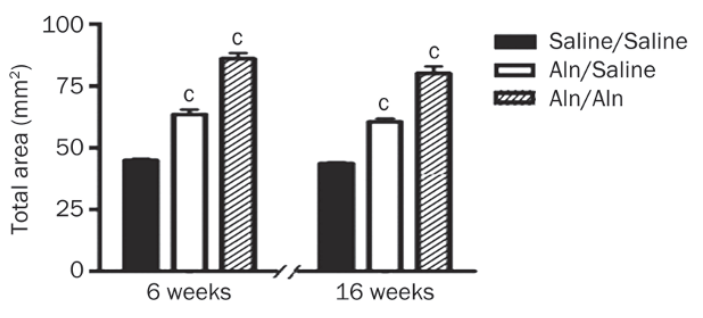

B

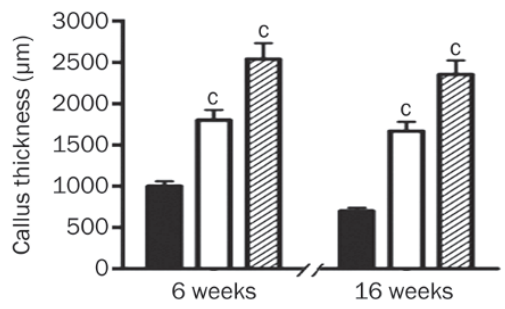

C

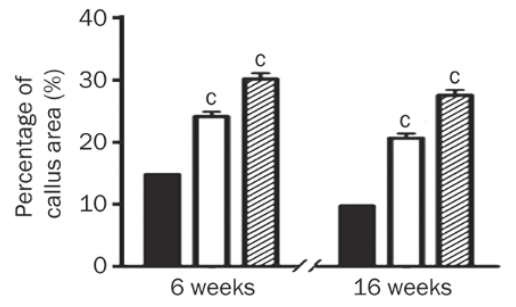

D

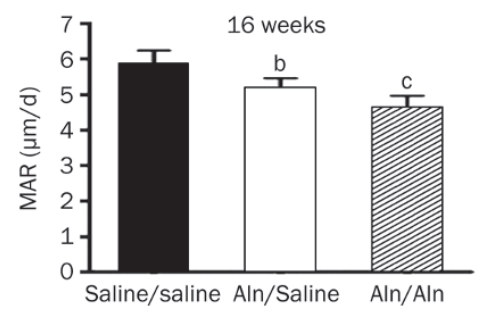

Figure 5. Quantitative analysis of the fracture calluses using $\mathrm{HE}$ staining ( $A, B$, and $C$ ) and fluorescence labeling (D). The data are expressed as the mean \pm SD $\left({ }^{b} P<0.05,{ }^{c} P<0.01\right.$ vs Saline/Saline, $\left.n=5\right)$. MAR: mineral apposition rate.

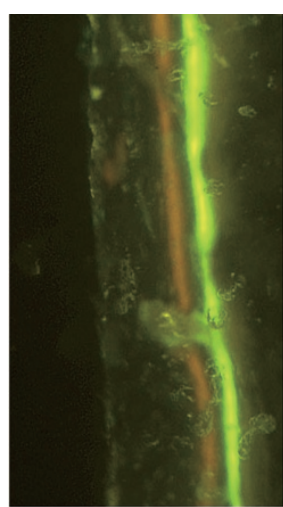

Saline/Saline

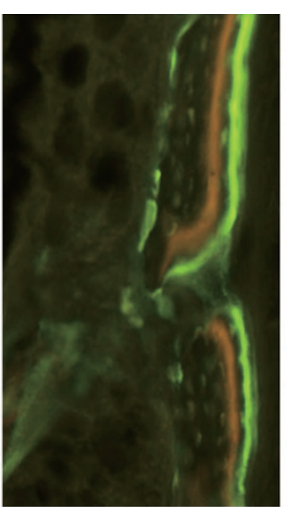

Aln/Saline

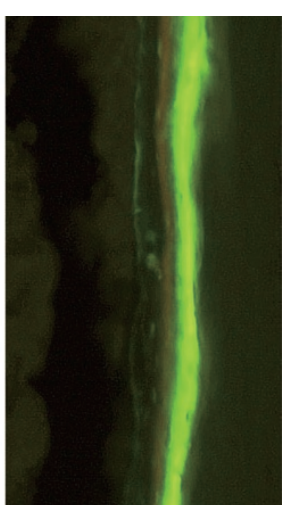

Aln/Aln
Figure 6. Representative fluorescence images of the fracture calluses at 16 weeks post-fracture. 


\section{Discussion}

Histology and mechanical tests have shown that osteoporotic fracture healing is a closely regulated process of restoring the structural geometry and mechanical properties of the broken bone ${ }^{[16-18]}$. In addition, it is well recognized that mechanical restoration of the fractured bone is the primary goal of fracture healing in the clinic; however, this mechanical restoration is not the ultimate completion of the fracture healing process. Geometrical and histological restorations are also essential for the completion of the natural fracture healing process.

In this study, we assessed the long-term effect of Aln on the biomechanical properties and the microstructure of the bony callus in ovariectomized rats. According to the threepoint bending test, the mechanical parameters of the callus in the Aln groups were higher than the control group. However, the microstructure of the callus in the Aln groups, as visualized by HE staining, was inferior to that of the control group. The subsequent quantitative analysis showed that T.Ar, Cl.Th and percentage callus area in the Aln groups were higher compared with the Saline/Saline group. The dynamic remodeling parameter, MAR, was suppressed with Aln treatment. This effect was more evident in the Aln/Aln group because of Aln treatment post-fracture. Therefore, these data suggest that Aln is beneficial for the mechanical properties of the callus but delays callus remodeling by suppressing the remodeling of woven bone into lamellar bone.

It is widely accepted that BP function in vivo as anti-catabolic agents, although several cell-based studies have shown an anabolic effect in vitro. BP are now well established as effective therapeutic agents for a variety of bone diseases ${ }^{[19]}$. Previously, a study using growing rats by Koivukangas et al showed that 24 weeks of clodronate (dichloromethylene bisphosphonate) pretreatment increased the size of the tibiae callus but had only a minor effect on its biomechanical properties, suggesting that clodronate treatment does not prolong the fracture healing process, even when administered on a longterm basis before fracture ${ }^{[20]}$. In addition, another study, also conducted in growing rats, evaluated the effects of incadronate disodium (YM-175) on fracture healing of the femoral shaft by dosing incadronate 2 weeks before fracture and 25 or 49 weeks after surgery. The results showed that long-term continuous treatment with incadronate delayed the process of fracture healing in the femur in rats, especially under a high dose, but did not impair the recovery of the mechanical integrity of the fracture $^{[14]}$. Based on these results and our own, we conclude that BP may not impair or improve the mechanical properties of the callus but delay the histological recovery.

There are several limitations of this study. First, our fracture model was established by surgical osteotomy, which differs from a closed fracture model, which is similar to common clinical fractures. However, this procedure enabled us to make a consistent fracture line, which is essential for reliable and accurate evaluation. Second, a three-point bending test was used to evaluate the fracture callus. In this test, the indenter is put over the central part of the callus, and the resulting mea- surement depends in part on the material properties where the indenter is put. A four-point bending test or a torsion test would be better for the fracture calluses in our future study.

In summary, the current study provided evidence that long-term Aln therapy before fracture would favor the formation of a mechanically competent large callus with a slow rate of remodeling in an ovariectomized rat model. Continued Aln treatment post-fracture would further aggravate this effect.

\section{Acknowledgements}

This work was supported by the Key Project of the Science and Technology Commission of Shanghai Municipality (Grant No 05JC14034), the Selecting and Training Outstanding Young Teachers in Shanghai Universities (Grant No jdy08068), the National Natural Science Foundation of China (Grant No 81201424), and the Program for Shanghai Key Laboratory of Orthopaedic Implant (Grant No 08DZ2230330).

\section{Author contribution}

Ke-rong DAI designed the research; Ling-jie FU performed the research and analyzed the data; Ting-ting TANG contributed analytic tools; Ling-jie FU and Yong-qiang HAO wrote the paper.

\section{References}

1 Giannoudis P, Tzioupis C, Almalki T, Buckley R. Fracture healing in osteoporotic fractures: is it really different? A basic science perspective. Injury 2007; 38 Suppl 1: S90-9.

2 Geusens P. Bisphosphonates for postmenopausal osteoporosis: determining duration of treatment. Curr Osteoporos Rep 2009; 7: 12-7.

3 Müller D, Pulm J, Gandjour A. Cost-effectiveness of different strategies for selecting and treating individuals at increased risk of osteoporosis or osteopenia: a systematic review. Value Health 2012; 15: $284-98$

4 Fu L, Tang T, Miao Y, Zhang S, Qu Z, Dai K. Stimulation of osteogenic differentiation and inhibition of adipogenic differentiation in bone marrow stromal cells by alendronate via ERK and JNK activation. Bone 2008; 43: 40-7.

5 Schneider JP. Bisphosphonates and low-impact femoral fractures: current evidence on alendronate-fracture risk. Geriatrics 2009; 64: 18-23.

6 Shin DY, Ku CR, Kim KM, Choi HS, Rhee Y, Lee EJ, et al. Spontaneous non-traumatic stress fractures in bilateral femoral shafts in a patient treated with bisphosphonates. Korean J Intern Med 2012; 27: 98-102.

7 Yang KH, Won JH, Yoon HK, Ryu JH, Choo KS, Kim JS. High concentrations of pamidronate in bone weaken the mechanical properties of intact femora in a rat model. Yonsei Med J 2007; 48: 653-8.

8 Chapurlat RD, Delmas PD. Drug insight: Bisphosphonates for postmenopausal osteoporosis. Nat Clin Pract Endocrinol Metab 2006; 2: 211-9; quiz following 238.

9 Sliwinski L, Janiec W, Pytlik M, Folwarczna J, Kaczmarczyk-Sedlak I, Pytlik W, et al. Effect of administration of alendronate sodium and retinol on the mechanical properties of the femur in ovariectomized rats. Pol J Pharmacol 2004; 56: 817-24.

10 Li C, Mori S, Li J, Kaji Y, Akiyama T, Kawanishi J, et al. Long-term effect of incadronate disodium (YM-175) on fracture healing of femoral shaft in growing rats. J Bone Miner Res 2001; 16: 429- 
36.

11 Cao Y, Mori S, Mashiba T, Westmore MS, Ma L, Sato M, et al. Raloxifene, estrogen, and alendronate affect the processes of fracture repair differently in ovariectomized rats. J Bone Miner Res 2002; 17: 2237-46.

12 Komatsubara S, Mori S, Mashiba T, Nonaka K, Seki A, Akiyama T, et al. Human parathyroid hormone (1-34) accelerates the fracture healing process of woven to lamellar bone replacement and new cortical shell formation in rat femora. Bone 2005; 36: 678-87.

13 Li J, Mori S, Kaji Y, Kawanishi J, Akiyama T, Norimatsu H. Concentration of bisphosphonate (incadronate) in callus area and its effects on fracture healing in rats. J Bone Miner Res 2000; 15 : 2042-51.

14 Li J, Mori S, Kaji Y, Mashiba T, Kawanishi J, Norimatsu H. Effect of bisphosphonate (incadronate) on fracture healing of long bones in rats. J Bone Miner Res 1999; 14: 969-79.

15 Li H, Dai K, Tang T, Zhang X, Yan M, Lou J. Bone regeneration by implantation of adipose-derived stromal cells expressing BMP-2.
Biochem Biophys Res Commun 2007; 356: 836-42.

16 Wang JW, Li W, Xu SW, Yang DS, Wang Y, Lin M, et al. Osteoporosis influences the middle and late periods of fracture healing in a rat osteoporotic model. Chin J Traumatol 2005; 8: 111-6.

17 Kubo T, Shiga T, Hashimoto J, Yoshioka M, Honjo H, Urabe M, et al. Osteoporosis influences the late period of fracture healing in a rat model prepared by ovariectomy and low calcium diet. J Steroid Biochem Mol Biol 1999; 68: 197-202.

18 Namkung-Matthai H, Appleyard R, Jansen J, Hao Lin J, Maastricht S, Swain $\mathrm{M}$, et al. Osteoporosis influences the early period of fracture healing in a rat osteoporotic model. Bone 2001; 28: 80-6.

19 Koester MC, Spindler KP. Pharmacologic agents in fracture healing. Clin Sports Med 2006; 25: 63-73, viii.

20 Koivukangas A, Tuukkanen J, Kippo K, Jämsä T, Hannuniemi R, Pasanen I, et al. Long-term administration of clodronate does not prevent fracture healing in rats. Clin Orthop Relat Res 2003; 408: 268-78. 